\title{
A wave equation with structural damping and nonlinear memory
}

\author{
Marcello D'Abbicco
}

\begin{abstract}
In this paper, we obtain the critical exponent for a wave equation with structural damping and nonlinear memory:

$$
u_{t t}-\triangle u+\mu(-\triangle)^{\frac{1}{2}} u_{t}=\int_{0}^{t}(t-s)^{-\gamma}|u(s, \cdot)|^{p} d s,
$$

where $\mu>0$. In the supercritical case, we prove the existence of small data global solutions, whereas, in the subcritical case, we prove the nonexistence of global solutions for suitable arbitrarily small data, in the special case $\mu=2$.
\end{abstract}

Mathematics Subject Classification (2010). 35L71.

Keywords. Semilinear equations, Global existence, Nonlinear memory, Structural damping, Critical exponent.

\section{Introduction}

We study the global existence of small data solutions to

$$
\left\{\begin{array}{l}
u_{t t}-\triangle u+\mu(-\triangle)^{\frac{1}{2}} u_{t}=F(t, u), \\
u(0, x)=u_{0}(x), \\
u_{t}(0, x)=u_{1}(x),
\end{array}\right.
$$

where $\mu>0$, and the term

$$
F(t, u):=\int_{0}^{t}(t-s)^{-\gamma}|u(s, \cdot)|^{p} d s,
$$

for some $\gamma \in(0,1)$ and $p>1$, represents a nonlinear memory.

Recently, fractional PDEs have been deeply investigated, since they are particularly interesting for the real world applications. According to [23], fractional derivatives provide an excellent instrument for the description of memory and hereditary process. The advantage of fractional derivatives become 
apparent in modelling mechanical and electrical properties of real materials and in many other fields.

In particular, the linear part of the equation in (1.1), i.e.

$$
u_{t t}-\triangle u+\mu(-\triangle)^{\frac{1}{2}} u_{t}=0,
$$

is frequently used in the determination of lifespan for primary or rechargeable batteries [10]. The model in (1.3) is a special case of a wave equation with structural damping:

$$
u_{t t}-\triangle u+\mu(-\triangle)^{\sigma} u_{t}=0, \quad \sigma>0 .
$$

Different kind of estimates in Sobolev spaces for (1.4) with $\sigma \in(0,1)$ have been recently studied in $[1,14,22]$. In the limit case $\sigma=1$, the damping is also called visco-elastic and it has been studied in $[18,26]$ and, in abstract setting, in $[13,16]$. Some $L^{2}-L^{2}$ estimates have been derived for (1.4) in the case with time-dependent damping $b(t)(-\triangle)^{\sigma} u_{t}$ in $[19,24]$. Smoothing effects for (1.4) are studied in [12], including the case $\sigma \geq 1$.

The nonlinear term $F(t, u)$ in (1.2) may be written as

$$
F(t, u)=\Gamma(1-\gamma) J_{0 \mid t}^{1-\gamma}\left(|u|^{p}\right),
$$

where $\Gamma$ is the Euler Gamma function, and $J_{0 \mid t}^{1-\gamma}\left(|u|^{p}\right)$ is the fractional Riemann-Liouville integral of $|u(\tau, \cdot)|^{p}$ in $[0, t]$. Therefore, it is reasonable to expect relations with the case of a power nonlinearity $F(u)=|u|^{p}$, as $\gamma \rightarrow 1$. It has been recently proved [8] that small data global solutions to

$$
\left\{\begin{array}{l}
u_{t t}-\triangle u+\mu(-\triangle)^{\frac{1}{2}} u_{t}=|u|^{p}, \\
u(0, x)=u_{0}(x), \\
u_{t}(0, x)=u_{1}(x),
\end{array}\right.
$$

exist if $p>1+2 /(n-1)$, and that this exponent is optimal. On the other hand, the optimal critical exponent for global small data solutions to

$$
\left\{\begin{array}{l}
u_{t t}-\triangle u+\mu u_{t}=F(t, u), \\
u(0, x)=u_{0}(x) \\
u_{t}(0, x)=u_{1}(x)
\end{array}\right.
$$

is given by (see $[4,11]$ )

$$
\max \left\{\tilde{p}_{\gamma}(n), \gamma^{-1}\right\}, \quad \tilde{p}_{\gamma}(n):=1+\frac{2(2-\gamma)}{[n-2(1-\gamma)]_{+}} .
$$

From the point of view of the global existence argument, the transition from the exponent $\tilde{p}_{\gamma}(n)$ to the exponent $\gamma^{-1}$, which occurs at $\gamma=(n-2) / n$, is deeply related to the profile of the decay estimates for the solution to (1.6).

It is natural to ask in which way the critical exponent is influenced if we replace the power nonlinearity in (1.5) by a nonlinear memory term.

The purpose of this paper is to give an answer to this question. We study into details the model in (1.3), stating only partial results for the general case (1.4) (see Sect. 5), for two reasons. 
- The special structure of (1.3) allows us to derive a global existence result for (1.1) in any space dimension $n \geq 2$ (see later, Remark 3.1).

- It is possible to use the test function method to prove a counterpart result of nonexistence of global solutions thanks to a maximum principle (see later, Lemma 2.10).

We will prove that the critical exponent for (1.1) is

$$
\bar{p}(n, \gamma)=\max \left\{p_{\gamma}(n), \gamma^{-1}\right\}, \quad p_{\gamma}(n):=1+\frac{3-\gamma}{n+\gamma-2},
$$

for any $n \geq 2$. We notice that

$$
\bar{p}(2, \gamma)=p_{\gamma}(2)=3 \gamma^{-1}
$$

whereas for any $n \geq 3$ it holds

$$
\bar{p}(n, \gamma)= \begin{cases}p_{\gamma}(n) & \text { if }(n-2) / n \leq \gamma<1, \\ \gamma^{-1} & \text { if } 0<\gamma \leq(n-2) / n .\end{cases}
$$

In particular, the threshold $(n-2) / n$ for $\gamma$, which denotes the transition to the critical exponent $\gamma^{-1}$ is the same for both models (1.1) and (1.6). We notice that $\tilde{p}_{\gamma}(n)<p_{\gamma}(n)$ if, and only if, $\gamma \in((n-2) / n, 1)$. This suggests that if the nonlinear memory term is sufficiently strong in space dimension $n \geq 3$, namely $\gamma \in(0,(n-2) / n]$, then the influence from classical and structural damping is the same. Otherwise, the critical exponent is larger in the structural damping case, as it happens for the power nonlinearity $|u|^{p}$.

\section{Results}

In each statement of this paper, we will briefly denote the normed space of initial data of the Cauchy problem as $\mathcal{A}$. Therefore, the definition of $\mathcal{A}$ will be related to the statement in which it appears. By the letter $\varepsilon>0$ we will denote the smallness of initial data in the norm of $\mathcal{A}$. On the other hand, in the following, we will respectively denote sufficiently small and sufficiently large positive constants by $\epsilon>0$ and $C>0$.

In the following, we generalize the nonlinear term in (1.2) to

$$
F(t, u)=\int_{0}^{t}(t-s)^{-\gamma} g(u(s, \cdot)) d s,
$$

where $g: \mathbb{R} \rightarrow \mathbb{R}$ is a locally Lipsichitz function satisfying

$$
g(0)=0, \quad|g(u)-g(v)| \lesssim|u-v|\left(|u|^{p-1}+|v|^{p-1}\right), \quad \text { for some } p>1 .
$$

Definition 2.1. For any $q \in[1, \infty)$, we define $q^{\sharp}=q^{\sharp}(n, q)$ as

$$
q^{\sharp}:=\max \left\{\frac{n q}{n+q}, 1\right\} \text {. }
$$

If $q=\infty$, we put $\infty^{\sharp}=n$.

We notice that $q^{\sharp}$ is the Sobolev conjugate of $q$ if $q \geq n /(n-1)$. 
Definition 2.2. For any function $u \in \mathcal{C}\left([0, T], H^{1}\right) \cap \mathcal{C}^{1}\left([0, T], L^{2}\right)$ we define

$$
\mathcal{E}[u](t):=\|\nabla u(t, \cdot)\|_{L^{2}}+\left\|u_{t}(t, \cdot)\right\|_{L^{2}},
$$

which is equivalent to the square root of the classical energy of $u$.

We are now ready to state our results. For the sake of clarity, we first deal with space dimension $n \geq 3$.

Theorem 2.3. Let $n \geq 3, \gamma \in(0,1)$ and $p>\bar{p}(n, \gamma)$. Let

$$
r \geq \max \left\{n(p-1), \frac{2 n}{n-2}\right\},
$$

and $r^{\sharp}$ be defined as in (2.3). Then there exists $\varepsilon>0$ such that for any initial data

$$
\left(u_{0}, u_{1}\right) \in \mathcal{A}:=\left(L^{1} \cap H^{1} \cap L^{r}\right) \times\left(L^{1} \cap L^{r^{\sharp}}\right) \quad \text { with }\left\|\left(u_{0}, u_{1}\right)\right\|_{\mathcal{A}} \leq \varepsilon,
$$

there exists a $\mathcal{C}\left([0, \infty), L^{1} \cap H^{1} \cap L^{r}\right) \cap \mathcal{C}^{1}\left([0, \infty), L^{2}\right)$ solution to (1.1). Moreover, the solution satisfies the estimates

$$
\begin{gathered}
\|u(t, \cdot)\|_{L^{q}} \leq \begin{cases}C(1+t)^{-\gamma+2-n\left(1-\frac{1}{q}\right)}\left\|\left(u_{0}, u_{1}\right)\right\|_{\mathcal{A}} & \text { if } q \in[1, n /(n-2)), \\
C(1+t)^{-\gamma} \ln (e+t)\left\|\left(u_{0}, u_{1}\right)\right\|_{\mathcal{A}} & \text { if } q=n /(n-2), \\
C(1+t)^{-\gamma}\left\|\left(u_{0}, u_{1}\right)\right\|_{\mathcal{A}} & \text { if } q \in(n /(n-2), r]\end{cases} \\
\mathcal{E}[u](t) \leq C(1+t)^{-\gamma}\left\|\left(u_{0}, u_{1}\right)\right\|_{\mathcal{A}}
\end{gathered}
$$

If we set $r=\infty$ in Theorem 2.7, we obtain a statement for any $p>\bar{p}(n, \gamma)$.

Remark 2.4. If (2.4) holds for some $p>1$ and $n \geq 3$, then it follows that, in particular, $r \geq 2 p$. Indeed, if $p \leq n /(n-2)$ the statement trivially follows from $r \geq 2 n /(n-2)$. Otherwise, if $p>n /(n-2)$ then it holds

$$
r \geq n(p-1)=2 p+(n-2) p-n>2 p .
$$

The bound $r \geq 2 n /(n-2)$ in Theorem 2.3 is a consequence of the assumption $u_{0} \in H^{1}$, since $H^{1} \subset L^{\frac{2 n}{n-2}}$ for $n \geq 3$. Indeed, if we set $\bar{r}=2 n /(n-2)$, then $\bar{r}^{\sharp}=2$ and we obtain the following.

Corollary 2.5. Let $n \geq 3, \gamma \in((n-2) / n, 1)$ and $p_{\gamma}(n)<p \leq n /(n-2)$. Then there exists $\varepsilon>0$ such that for any initial data

$$
\left(u_{0}, u_{1}\right) \in \mathcal{A}:=\left(L^{1} \cap H^{1}\right) \times\left(L^{1} \cap L^{2}\right) \text { with }\left\|\left(u_{0}, u_{1}\right)\right\|_{\mathcal{A}} \leq \varepsilon,
$$

there exists a $\mathcal{C}\left([0, \infty), L^{1} \cap H^{1}\right) \cap \mathcal{C}^{1}\left([0, \infty), L^{2}\right)$ solution to (1.1). Moreover, the solution satisfies estimates (2.6) with $r=2 n /(n-2)$, and estimate (2.7).

The restriction $p \leq n /(n-2)$ in Corollary 2.5 is equivalent to assumption (2.4) for $r=2 n /(n-2)$, in Theorem 2.3. Moreover, the restriction $p \leq n /(n-2)$ implies that the interval $(\bar{p}(n, \gamma), n /(n-2)]$ is nonempty if, and only if, $\gamma \in$ $((n-2) / n, 1)$. This gives $\bar{p}(n, \gamma)=p_{\gamma}(n)$.

If $n=2$, we have the following. 
Theorem 2.6. Let $n=2, \gamma \in(0,1)$ and $p>3 \gamma^{-1}$. Then there exists $\varepsilon>0$ such that for any initial data as in (2.8), there exists a $\mathcal{C}\left([0, \infty), L^{1} \cap H^{1}\right) \cap$ $\mathcal{C}^{1}\left([0, \infty), L^{2}\right)$ solution to (1.1). Moreover, the solution satisfies the estimates

$$
\begin{aligned}
\|u(t, \cdot)\|_{L^{q}} & \leq C(1+t)^{-\gamma+2 / q}\left\|\left(u_{0}, u_{1}\right)\right\|_{\mathcal{A}} \quad \text { for any } q \in[1, \infty), \\
\mathcal{E}[u](t) & \leq C(1+t)^{-\gamma} \ln (e+t)\left\|\left(u_{0}, u_{1}\right)\right\|_{\mathcal{A}} .
\end{aligned}
$$

If we also assume that $u_{0} \in L^{\infty}$ and $\left\|u_{0}\right\|_{L^{\infty}} \leq \varepsilon$, with $\varepsilon$ as in (2.8), then the solution $u$ is also continuous from $[0, \infty)$ into $L^{\infty}$ and it satisfies the following estimate:

$$
\|u(t, \cdot)\|_{L^{\infty}} \leq C(1+t)^{-\gamma} \ln (e+t)\left\|\left(u_{0}, u_{1}\right)\right\|_{\mathcal{A}} .
$$

If we are not interested in energy solutions, we may drop the assumption $u_{0} \in H^{1}$ in Theorem 2.3. In this case, we say that $u \in \mathcal{C}\left([0, \infty), L^{1} \cap L^{r}\right)$ is a global solution to (1.1) if it solves (1.1) in a weak sense. We may now state the following.

Theorem 2.7. Let $n \geq 2$ and $\gamma \in(0,1)$. Let $p>\bar{p}(n, \gamma), r \geq n(p-1)$ and $r^{\sharp}$ as in (2.3). Then there exists $\varepsilon>0$ such that for any initial data

$$
\left(u_{0}, u_{1}\right) \in \mathcal{A}:=\left(L^{1} \cap L^{r}\right) \times\left(L^{1} \cap L^{r^{\sharp}}\right) \quad \text { with }\left\|\left(u_{0}, u_{1}\right)\right\|_{\mathcal{A}} \leq \varepsilon,
$$

there exists a weak $\mathcal{C}\left([0, \infty), L^{1} \cap L^{r}\right)$ solution to (1.1). Moreover, the solution satisfies estimate (2.6) if $n \geq 3$, and estimate

$$
\begin{gathered}
\|u(t, \cdot)\|_{L^{q}} \leq C(1+t)^{-\gamma+2 / q}\left\|\left(u_{0}, u_{1}\right)\right\|_{\mathcal{A}} \quad \text { for any } q \in(1, r], \quad \text { (2.13) } \\
\text { if } n=2 \text { and } r<\infty \text {, or estimates }(2.9) \text { and }(2.11) \text {, if } n=2 \text { and } r=\infty \text {. }
\end{gathered}
$$

The Sobolev exponent $r$ in Theorem 2.7 may be now lesser than $2 n /(n-$ $2)$, since we dropped the hypothesis $u_{0} \in H^{1}$. However, restriction $r \geq n(p-1)$ implies that $r>2 n /(n-1)$ for any $\gamma \in(0,1)$ and $p>\bar{p}(n, \gamma)$. In particular, the interval $(n /(n-2), r]$ in estimate (2.6) is never empty if $n \geq 3$.

Remark 2.8. Assumptions $r \geq n(p-1)$ and $p>\bar{p}(n, \gamma)$ imply that $r>p+1$ for any $\gamma \in(0,1)$. Indeed, $\bar{p}(n, \gamma)>1+2 /(n-1)$ for any $\gamma \in(0,1)$, hence

$$
r \geq p-1+(n-1)(p-1)>p-1+(n-1)(\bar{p}(n, \gamma)-1)>p+1 .
$$

Remark 2.9. In estimates (2.6), (2.7), (2.9), (2.10), (2.11) and (2.13) it does not appear a decay rate better than $(1+t)^{-\gamma}$. This restriction comes from the influence of the nonlinear memory term (see Lemma 3.2). In particular, if $q<n /(n-2)$, a loss of decay $(1+t)^{1-\gamma}$ with respect to the corresponding linear estimates [see (3.3)] appears in estimate (2.6). This loss is larger if $q>n /(n-2)$. This phenomenon has been described in [4].

\subsection{Optimality of the critical exponent}

The fractional Laplacian is a nonlocal operator, thus it is not easy, in general, to prove the optimality of $\bar{p}(n, \gamma)$, by using the test function method. We are able to do that if $\mu=2$ and $u_{0} \equiv 0$ in (1.1), thanks to a maximum principle for $(1.3)$. 
Lemma 2.10. The solution to

$$
\left\{\begin{array}{l}
u_{t t}-\triangle u+2(-\triangle)^{\frac{1}{2}} u_{t}=0 \\
u(0, x)=0 \\
u_{t}(0, x)=u_{1}(x) \geq 0
\end{array}\right.
$$

is nonnegative, and it is explicitly given by

$$
u(t, x)=c_{n} \frac{t}{\left(|x|^{2}+t^{2}\right)^{\frac{n+1}{2}}} *(x) u_{1}(x),
$$

where $c_{n}$ depends on the space dimension [22].

Thanks to Lemma 2.10, we can state the following.

Theorem 2.11. Let us consider the Cauchy problem

$$
\left\{\begin{array}{l}
u_{t t}-\Delta u+2(-\triangle)^{\frac{1}{2}} u_{t}=\int_{0}^{t}(t-s)^{-\gamma} u(s, x)^{p} d s \\
u(0, x)=0 \\
u_{t}(0, x)=u_{1}(x) \geq 0
\end{array}\right.
$$

with $\gamma \in(0,1), p>1$ and $u_{1} \in L_{\text {loc }}^{1}$, non trivial. Then there exists no global weak solution to (2.15) in the following four cases:

- if $n=1$, for any $\gamma \in(0,1)$ and $p>1$,

- if $n \geq 2$, for any $\gamma \in[(n-2) / n, 1)$ and $p \in\left(1, p_{\gamma}(n)\right]$,

- if $n \geq 3$, for any $\gamma \in(0,(n-2) / n)$ and $p \in\left(1, \gamma^{-1}\right)$.

In view of the previously stated results of global existence, Theorem 2.11 says that the critical exponent $\bar{p}(n, \gamma)$ is optimal, in general. It remains open to study what happens if $\gamma \in(0,(n-2) / n)$ and $p=\gamma^{-1}$, since this case is not included in our global existence statements, neither in Theorem 2.11.

\section{Proof of the global existence results}

In [22] it has been proved that the solution to the linear problem

$$
\left\{\begin{array}{l}
v_{t t}-\triangle v+\mu(-\triangle)^{\frac{1}{2}} v_{t}=0 \\
v(0, x)=v_{0}(x) \\
v_{t}(0, x)=v_{1}(x)
\end{array}\right.
$$

satisfies the estimate

$$
\|v(t, \cdot)\|_{L^{q}} \leq t^{-n\left(\frac{1}{q_{0}}-\frac{1}{q}\right)}\left\|v_{0}\right\|_{L^{q_{0}}}+t^{1-n\left(\frac{1}{q_{1}}-\frac{1}{q}\right)}\left\|v_{1}\right\|_{L^{q_{1}}},
$$

for any $q_{0}, q_{1} \geq 1$ and $q \geq \max \left\{q_{0}, q_{1}\right\}$. In particular, setting $q_{0}=q_{1}=1$ for $t \geq 1$, and $q_{0}=q, q_{1}=q^{\sharp}$ for $t \in[0,1]$, we may derive

$$
\|v(t, \cdot)\|_{L^{q}} \leq(1+t)^{1-n\left(1-\frac{1}{q}\right)}\left(\left\|v_{0}\right\|_{L^{1} \cap L^{q}}+\left\|v_{1}\right\|_{L^{1} \cap L^{q^{\sharp}}}\right),
$$

where $q^{\sharp}$ is as in (2.3). Indeed,

$$
1-n\left(\frac{1}{q^{\sharp}}-\frac{1}{q}\right) \geq 1-n\left(\frac{n+q}{n q}-\frac{1}{q}\right)=0 .
$$


The solution to (3.1) satisfies the energy estimate (see, for instance, [8]):

$$
\mathcal{E}[v](t) \leq(1+t)^{-\frac{n}{2}}\left(\left\|v_{0}\right\|_{L^{1} \cap H^{1}}+\left\|v_{1}\right\|_{L^{1} \cap L^{2}}\right) .
$$

Remark 3.1. An important difference between linear estimates (3.2) for (1.1) and the corresponding linear estimates for (1.6) is that, in this latter case, a loss of regularity appears in the estimate for $\|u(t, \cdot)\|_{L^{q}}$ when $q \neq 2$ (see, for instance, [21]). This loss of regularity increases as the space dimension $n$ grows, imposing an heavy restriction to the applicability of these estimates to prove global existence in high space dimension. This restriction does not appear in (1.1), so we may obtain a global existence result in any space dimension $n \geq 2$, and this motivated us to focus on this model.

We remark that in the case of the classical damped wave equation with power nonlinearity $|u|^{p}$ this problem has been solved in $[15,27]$ by using weighted energy estimates for the nonlinear problem.

A fundamental role will be played by the following.

Lemma 3.2. Let $\alpha \in \mathbb{R}, \beta>1$ and $\gamma \in(0,1)$. Then it holds

$$
\int_{0}^{t}(1+t-\tau)^{-\alpha} \int_{0}^{\tau}(\tau-s)^{-\gamma}(1+s)^{-\beta} d s d \tau \lesssim \begin{cases}(1+t)^{1-\alpha-\gamma} & \text { if } \alpha<1, \\ (1+t)^{-\gamma} \ell(t) & \text { if } \alpha=1, \\ (1+t)^{-\gamma} & \text { if } \alpha>1,\end{cases}
$$

where $\ell(t):=\ln (e+t)$.

Proof. First let us consider the interior integral. Since $\gamma \in(0,1)$ and $\beta>1$, we may estimate (see, for instance, Lemma 4.1 in [3])

$$
\int_{0}^{\tau}(\tau-s)^{-\gamma}(1+s)^{-\beta} d s \lesssim(1+\tau)^{-\gamma}
$$

Therefore,

$\int_{0}^{t}(1+t-\tau)^{-\alpha} \int_{0}^{\tau}(\tau-s)^{-\gamma}(1+s)^{-\beta} d s d \tau \lesssim \int_{0}^{t}(1+t-\tau)^{-\alpha}(1+\tau)^{-\gamma} d \tau$

and the proof immediately follows (see, for instance, $[9,20]$ ).

We also recall Gagliardo-Nirenberg inequality:

$$
\|u\|_{L^{q}} \leq C\|u\|_{L^{2}}^{1-\eta}\|\nabla u\|_{L^{2}}^{\eta}, \quad \eta=n\left(\frac{1}{2}-\frac{1}{q}\right),
$$

for any $q \in[2, \infty]$ if $n=1$, for any $q \in[2, \infty)$ if $n=2$, and for any $q \in[2,2 n /(n-2)]$ if $n \geq 3$.

We are now ready to prove our statements.

Proof of Theorem 2.3. For any $T>0$, we introduce the space

$$
X(T):=\mathcal{C}\left([0, T], L^{1} \cap H^{1} \cap L^{r}\right) \cap \mathcal{C}^{1}\left([0, T], L^{2}\right),
$$


with norm given by

$$
\begin{aligned}
\|w\|_{X(T)}:= & \max _{t \in[0, T]}\left\{(1+t)^{-(2-\gamma)}\|w(t, \cdot)\|_{L^{1}}\right. \\
& \left.+(1+t)^{\gamma}\left(\ell(t)^{-1}\|w(t, \cdot)\|_{L^{\bar{q}}}+\|w(t, \cdot)\|_{L^{r}}+\mathcal{E}[w](t)\right)\right\},
\end{aligned}
$$

where

$$
\bar{q}=\bar{q}(n):=\frac{n}{n-2},
$$

and $\ell(t):=\ln (e+t)$ here and in the following. If $w \in X(T)$ then we derive, by interpolation, that

$$
\|w(t, \cdot)\|_{L^{q}} \leq(1+t)^{-\gamma+[2-n(1-1 / q)]^{+}} \ell(t)\|w\|_{X(T)}, \quad \text { for any } q \in[1, r] .
$$

We consider the operator $N$ defined by

$$
N w:=u^{\operatorname{lin}}+G w, \quad \text { with } G w:=\int_{0}^{t} E(t-\tau, x) *_{(x)} F(\tau, w) d \tau,
$$

where $u^{\text {lin }}(t, x)$ is the solution to (3.1) with $\left(v_{0}, v_{1}\right)=\left(u_{0}, u_{1}\right)$, and $E(t, x)$ is the fundamental solution to (3.1) for $v_{0} \equiv 0$ and $v_{1}=\delta$. A function $w \in X(T)$ is a solution to (1.1) for any $t \in[0, T]$ if, and only if, $w=N w$ in $X(T)$. If we prove that

$$
\begin{aligned}
\|N w\|_{X(T)} & \leq C_{1}\left\|\left(u_{0}, u_{1}\right)\right\|_{\mathcal{A}}+C_{2}\|w\|_{X(T)}^{p}, \\
\|N w-N \tilde{w}\|_{X(T)} & \leq C\|w-\tilde{w}\|_{X(T)}\left(\|w\|_{X(T)}^{p-1}+\|\tilde{w}\|_{X(T)}^{p-1}\right),
\end{aligned}
$$

where $C_{1}, C_{2}$ and $C$ do not depend on $T$, by standard arguments (see, for instance, [7]), we may derive the existence of a unique fixed point of $N$ in $X(T)$, and then the existence of small data global solutions to (1.1), satisfying

$$
\|u\|_{X(T)} \leq \tilde{C}\left\|\left(u_{0}, u_{1}\right)\right\|_{\mathcal{A}}, \quad \text { uniformly for any } T>0 .
$$

Recalling the definition of $\mathcal{A}$ and setting $u_{0}=v_{0}$ and $u_{1}=v_{1}$ in estimates (3.3) and (3.4), we immediately derive that

$$
\left\|u^{\operatorname{lin}}\right\|_{X(T)} \leq C_{1}\left\|\left(u_{0}, u_{1}\right)\right\|_{\mathcal{A}}
$$

thus (3.11) follows once we prove that

$$
\|G w\|_{X(T)} \leq C\|w\|_{X(T)}^{p} .
$$

On the other hand, it is clear that we may rewrite (3.12) as

$$
\|G w-G \tilde{w}\|_{X(T)} \leq C\|w-\tilde{w}\|_{X(T)}\left(\|w\|_{X(T)}^{p-1}+\|\tilde{w}\|_{X(T)}^{p-1}\right) .
$$

We first prove (3.14). Recalling the definition of the operator $G$ in (3.10) and (2.2), thanks to (3.3) and (3.4), we obtain 


$$
\begin{aligned}
\|G w(t, \cdot)\|_{L^{q}} & \leq C \int_{0}^{t}(1+t-\tau)^{1-n\left(1-\frac{1}{q}\right)}\|F(\tau, w)\|_{L^{1} \cap L^{q^{\sharp}}} d \tau \\
& \leq C_{1} \int_{0}^{t}(1+t-\tau)^{1-n\left(1-\frac{1}{q}\right)} \int_{0}^{\tau}(\tau-s)^{-\gamma}\|w(\tau, \cdot)\|_{L^{p} \cap L^{p q^{\sharp}}}^{p} d s d \tau \\
\mathcal{E}[G w](t) & \leq C \int_{0}^{t}(1+t-\tau)^{-\frac{n}{2}}\|F(\tau, w)\|_{L^{1} \cap L^{2}} d \tau \\
& \leq C_{1} \int_{0}^{t}(1+t-\tau)^{-\frac{n}{2}} \int_{0}^{\tau}(\tau-s)^{-\gamma}\|w(\tau, \cdot)\|_{L^{p} \cap L^{2 p}}^{p} d s d \tau .
\end{aligned}
$$

We can set $q=p$ in (3.9) (see Remark 2.4), obtaining

$$
\|w(t, \cdot)\|_{L^{p}} \leq(1+t)^{-\gamma+[2-n(1-1 / p)]^{+}} \ell(t)\|w\|_{X(T)} .
$$

If, and only if, $p>\bar{p}(n, \gamma)$, then from the estimate above we may deduce that there exists $\beta(n, \gamma, p)>1$ such that

$$
\|w(t, \cdot)\|_{L^{p}}^{p} \leq C(1+t)^{-\beta(n, \gamma, p)}\|w\|_{X(T)}^{p},
$$

where $C$ is independent of $t$ and $T$. Having in mind (3.9), we see that $\|w(t, \cdot)\|_{L^{p q^{\sharp}}}^{p}$ also satisfies (3.16), provided that $p q^{\sharp} \leq r$,

for any $q \in[1, r]$. This latter holds if, and only if, $r \geq n(p-1)$.

Similarly, $\|w(t, \cdot)\|_{L^{2 p}}^{p}$ also satisfies (3.16) due to $2 p \leq r$ (see Remark 2.4).

We may now apply Lemma 3.2, since

$$
\begin{gathered}
\|G w(t, \cdot)\|_{L^{q}} \leq C\|w\|_{X(T)}^{p} \int_{0}^{t}(1+t-\tau)^{1-n\left(1-\frac{1}{q}\right)} I_{\gamma}(\tau) d \tau, \\
\mathcal{E}[G w](t) \leq C\|w\|_{X(T)}^{p} \int_{0}^{t}(1+t-\tau)^{-\frac{n}{2}} I_{\gamma}(\tau) d \tau
\end{gathered}
$$

where $I_{\gamma}(\tau)=\int_{0}^{\tau}(\tau-s)^{-\gamma}(1+s)^{-\beta} d s$,

for some $\beta>1$. Recalling that $1-n(1-1 / q)<1$ if, and only if, $q<\bar{q}(n)$, we obtain, in particular,

$$
\begin{aligned}
\|G w(t, \cdot)\|_{L^{1}} & \leq C(1+t)^{2-\gamma}\|w\|_{X(T)}^{p}, \\
\|G w(t, \cdot)\|_{L^{\bar{q}(n)}} & \leq C(1+t)^{-\gamma} \ell(t)\|w\|_{X(T)}^{p}, \\
\|G w(t, \cdot)\|_{L^{r}} & \leq C(1+t)^{-\gamma}\|w\|_{X(T)}^{p}, \\
\mathcal{E}[G w](t) & \leq C(1+t)^{-\gamma}\|w\|_{X(T)}^{p},
\end{aligned}
$$

and this concludes the proof of (3.14).

To prove (3.15), it is sufficient to use (2.2) and Hölder inequality, to estimate

$$
\|g(w)-g(\tilde{w})\|_{L^{1} \cap L^{q^{\sharp}}} \leq\|w-\tilde{w}\|_{L^{p} \cap L^{p q^{\sharp}}}\left(\|w\|_{L^{p} \cap L^{p q^{\sharp}}}^{p-1}+\|\tilde{w}\|_{L^{p} \cap L^{p q^{\sharp}}}^{p-1}\right),
$$

and similarly for $\|g(w)-g(\tilde{w})\|_{L^{1} \cap L^{2}}$, then we proceed as in the proof of

It remains to prove that the solution satisfies (2.6) and (2.7). For any fixed $t$ we may write $u=N u=u^{\text {lin }}+G u$. The estimates for $u^{\text {lin }}$ directly 
follows from $(3.3)-(3.4)$ with $\left(v_{0}, v_{1}\right)=\left(u_{0}, u_{1}\right)$. Therefore, it is sufficient to come back to (3.17) and to use again Lemma 3.2, to obtain

$$
\begin{aligned}
\|G u(t, \cdot)\|_{L^{q}} & \leq C\|u\|_{X(t)}^{p} \int_{0}^{t}(1+t-\tau)^{1-n\left(1-\frac{1}{q}\right)} I_{\gamma}(\tau) d \tau \\
& \leq C(1+t)^{-\gamma+[2-n(1-1 / q)]^{+}}\|u\|_{X(t)}^{p},
\end{aligned}
$$

for any $t \geq 0$ and $q \neq \bar{q}(n)$. The desired estimate follows thanks to (3.13) and the smallness of $\left\|\left(u_{0}, u_{1}\right)\right\|_{\mathcal{A}}$. Similarly, we derive the estimate in (2.6) for $q=\bar{q}(n)$ and estimate (2.7), taking into account of (3.20) and (3.22).

Proof of Theorem 2.6. We proceed as in the proof of Theorem 2.3, but we replace the definitions (3.6) and (3.7) of $X(T)$ and its norm by

$$
\begin{aligned}
X(T) & :=\mathcal{C}\left([0, T], L^{1} \cap H^{1}\right) \cap \mathcal{C}^{1}\left([0, T], L^{2}\right) \\
\|w\|_{X(T)} & :=\max _{t \in[0, T]}\left((1+t)^{-(2-\gamma)}\|w(t, \cdot)\|_{L^{1}}+(1+t)^{\gamma} \ell(t)^{-1} \mathcal{E}[w](t)\right) .
\end{aligned}
$$

By interpolation if $q \in[1,2]$, or by Gagliardo-Nirenberg inequality (3.5) if $q>2$, we obtain

$$
\|w(t, \cdot)\|_{L^{q}} \leq(1+t)^{-\gamma+2 / q} \ell(t)\|w\|_{X(T)}, \quad \text { for any } q \in[1, \infty) .
$$

This allows us to prove (3.14) and (3.15). In particular, it is clear that $p q^{\sharp}<\infty$ for any $q \in(1, \infty)$; hence, from $(3.25)$ we obtain that

$$
\|w(t, \cdot)\|_{L^{p} \cap L^{p q^{\sharp}}} \leq(1+t)^{-(n(1-1 / p)+\gamma-2)} \ell(t)\|w\|_{X(T)} .
$$

The global existence of the solution follows, then we prove the desired estimates, as in the proof of Theorem 2.3.

If we also assume $\left\|u_{0}\right\|_{L^{\infty}} \leq \varepsilon$, then we modify (3.23)-(3.24) accordingly,

$$
\begin{aligned}
X(T):= & \mathcal{C}\left([0, T], L^{1} \cap H^{1} \cap L^{\infty}\right) \cap \mathcal{C}^{1}\left([0, T], L^{2}\right) \\
\|w\|_{X(T)}:= & \max _{t \in[0, T]}\left\{(1+t)^{-(2-\gamma)}\|w(t, \cdot)\|_{L^{1}}\right. \\
& \left.+(1+t)^{\gamma} \ell(t)^{-1}\left(\|w(t, \cdot)\|_{L^{\infty}}+\mathcal{E}[w](t)\right)\right\}
\end{aligned}
$$

and we follow the proof of Theorem 2.3.

Proof of Theorem 2.7. The proof of Theorem 2.7 is analogous to the proof of Theorem 2.3, once we replace the definitions (3.6) and (3.7) of $X(T)$ and its norm by

$$
\begin{aligned}
X(T):= & \mathcal{C}\left([0, T], L^{1} \cap L^{r}\right) \\
\|w\|_{X(T)}:= & \max _{t \in[0, T]}\left\{(1+t)^{-(2-\gamma)}\|w(t, \cdot)\|_{L^{1}}\right. \\
& \left.+(1+t)^{\gamma} \ell(t)\right)^{-1}\|w(t, \cdot)\|_{L^{\bar{q}}} \\
& \left.+(1+t)^{\gamma}\|w(t, \cdot)\|_{L^{r}}\right\} \quad \text { if } n \geq 3
\end{aligned}
$$




$$
\begin{aligned}
\|w\|_{X(T)}:= & \max _{t \in[0, T]}\left\{(1+t)^{-(2-\gamma)}\|w(t, \cdot)\|_{L^{1}}\right. \\
& \left.+(1+t)^{\gamma}(\ell(t))^{-1}\|w(t, \cdot)\|_{L^{\infty}}\right\} \quad \text { if }(n, r)=(2, \infty), \\
\|w\|_{X(T)}:= & \max _{t \in[0, T]}\left\{(1+t)^{-(2-\gamma)}\|w(t, \cdot)\|_{L^{1}}\right. \\
& \left.+(1+t)^{\gamma-2 / r}\|w(t, \cdot)\|_{L^{r}}\right\} \quad \text { if } n=2 \text { and } r<\infty .
\end{aligned}
$$

The proof follows as in Theorem 2.3.

\section{Proof of Theorem 2.11}

Following [11], for any $\alpha \in(0,1)$ and for a fixed $T>0$, we introduce the fractional integral and differential operators $J_{0 \mid t}, D_{0 \mid t}, J_{t \mid T}, D_{t \mid T}$ defined by:

$$
\begin{aligned}
J_{0 \mid t}^{\alpha} f(t) & :=\frac{1}{\Gamma(\alpha)} \int_{0}^{t}(t-s)^{-(1-\alpha)} f(s) d s, \quad D_{0 \mid t}^{\alpha}:=\partial_{t} J_{0 \mid t}^{1-\alpha} \\
J_{t \mid T}^{\alpha} f(t) & :=\frac{1}{\Gamma(\alpha)} \int_{t}^{T}(s-t)^{-(1-\alpha)} f(s) d s, \quad D_{t \mid T}^{\alpha}:=-\partial_{t} J_{t \mid T}^{1-\alpha}
\end{aligned}
$$

We have the following properties (see (2.64), p. 46 in [25] and (2.106) in $[23])$ :

$$
\begin{aligned}
\int_{0}^{T}\left(D_{0 \mid t}^{\alpha} f\right)(t) g(t) d t & =\int_{0}^{T} f(t)\left(D_{t \mid T}^{\alpha} g\right)(t) d t \\
D_{0 \mid t}^{\alpha} J_{0 \mid t}^{\alpha} f(t) & =f(t) .
\end{aligned}
$$

Let us define

$$
\omega(t):= \begin{cases}(1-t / T) & \text { if } t \in[0, T] \\ 0 & \text { if } t>T .\end{cases}
$$

It follows that $\operatorname{supp} \omega=[0, T]$ and $\omega(t)^{\beta} \in \mathcal{C}_{c}^{k}([0, \infty)), k \geq 0$, for any $\beta>k$. Moreover, we have the following.

Lemma 4.1. For any $\alpha \in(0,1)$, it follows that

$$
D_{t \mid T}^{\alpha} \omega(t)^{\beta}=C(\alpha, \beta) T^{-\alpha} \omega(t)^{\beta-\alpha}, \quad \text { for any } \beta>\alpha,
$$

where

$$
C(\alpha, \beta)=\frac{\Gamma(\beta+1)}{(\beta+2-\alpha) \Gamma(\beta-\alpha)} .
$$

Proof. By the change of variables $\tau:=(s-t) /(T-t)$, we get (see [23])

$$
\begin{aligned}
\int_{t}^{T}(s-t)^{-\alpha}(1-t / T)^{\beta} d s & =(T-t)^{-\alpha+\beta+1} T^{-\beta} \int_{0}^{1} \tau^{-\alpha}(1-\tau)^{\beta} d \tau \\
& =\omega(t)^{-\alpha+\beta+1} T^{1-\alpha} \frac{\Gamma(1-\alpha) \Gamma(\beta+1)}{\Gamma(-\alpha+\beta+2)} .
\end{aligned}
$$


Therefore,

$$
\begin{aligned}
J_{t \mid T}^{1-\alpha} \omega(t)^{\beta} & =\frac{\Gamma(\beta+1)}{\Gamma(\beta+2-\alpha)} T^{1-\alpha} \omega(t)^{\beta+1-\alpha}, \\
D_{t \mid T}^{\alpha} \omega(t)^{\beta} & =\frac{\Gamma(\beta+1)}{(\beta+2-\alpha) \Gamma(\beta-\alpha)} T^{-\alpha} \omega(t)^{\beta-\alpha} .
\end{aligned}
$$

Now we are ready to prove Theorem 2.11.

Proof of Theorem 2.11. We put $\alpha:=1-\gamma$. By contradiction, let $u$ be a global weak solution to (1.1). Let $\Psi \in \mathcal{C}_{c}^{\infty}$ be a radial test function, such that:

- $\operatorname{supp} \Psi=B_{1}$;

- $\Psi(x)=1$, for any $x \in B_{1 / 2}$;

- $\Psi\left(x_{1}\right) \geq \Psi\left(x_{2}\right)$ if $\left|x_{1}\right| \leq\left|x_{2}\right|$.

For any $R \geq 1$, we denote $\Psi_{R}(t, x):=\Psi(x / R)$. Let us fix

$$
\beta>(\alpha+2) p^{\prime}, \text { and } \ell>p^{\prime},
$$

where $p^{\prime}:=p /(p-1)$ is the Hölder conjugate of $p$, and let

$$
\Phi_{R}(t, x):=\omega(t)^{\beta} \Psi_{R}(x)^{\ell}, \quad \varphi:=D_{t \mid T}^{\alpha} \Phi_{R}(t, x) .
$$

Then, $\operatorname{supp} \varphi \subset[0, T] \times B_{R}$, for any $T, R \geq 1$, but $\operatorname{supp}(-\triangle)^{\frac{1}{2}} \varphi \subset$ $[0, T] \times \mathbb{R}^{n}$, in general, since the fractional Laplacian is a nonlocal operator. We may write

$$
\begin{gathered}
\int_{0}^{T} \int_{B_{R}} u\left(\varphi_{t t}-\triangle \varphi\right) d x d t-2 \int_{0}^{T} \int_{\mathbb{R}^{n}} u(-\triangle)^{\frac{1}{2}} \varphi_{t} d x d t \\
\quad=\int_{B_{R}} u_{1}(x) \varphi(0, x) d x+\Gamma(\alpha) \int_{0}^{T} \int_{B_{R}} J_{0 \mid t}^{\alpha}\left(u^{p}\right) \varphi d x d t .
\end{gathered}
$$

We remark that $\varphi \geq 0$ thanks to (4.4). By virtue of (4.1) and (4.2), we get

$$
\int_{0}^{T} \int_{B_{R}} J_{0 \mid t}^{\alpha}\left(u^{p}\right) \varphi d x d t=\Gamma(\alpha) \int_{0}^{T} \int_{B_{R}} u^{p} \Phi_{R} d x d t
$$

whereas, being $u_{1}$ and $\varphi$ nonnegative, we obtain

$$
-\int_{B_{R}} u_{1}(x) \varphi(0, x) d x \leq 0 .
$$

Now we use Young inequality to estimate

$$
\int_{0}^{T} \int_{B_{R}} u \varphi_{t t} d x d t \leq \epsilon \int_{0}^{T} \int_{B_{R}} u^{p} \Phi_{R} d x d t+C_{\epsilon} \int_{0}^{T} \int_{B_{R}} \varphi_{t t} p^{p^{\prime}} \Phi_{R}^{-\frac{1}{p-1}} d x d t
$$

and, thanks to

$$
\begin{aligned}
\partial_{t}^{2} D_{t \mid T}^{\alpha} \omega(t)^{\beta} & =\frac{\Gamma(\beta+1)}{(\beta+2-\alpha) \Gamma(\beta-\alpha)} T^{-\alpha} \partial_{t}^{2} \omega(t)^{\beta-\alpha} \\
& =\frac{\Gamma(\beta+1)}{(\beta+2-\alpha) \Gamma(\beta-\alpha-2)} T^{-(\alpha+2)} \omega(t)^{\beta-\alpha-2},
\end{aligned}
$$


and

$$
\operatorname{meas}([0, T])=T, \quad \operatorname{meas}\left(B_{R}\right) \approx R^{n},
$$

we obtain

$$
\begin{aligned}
& \int_{0}^{T} \int_{B_{R}} \varphi_{t t^{p^{\prime}}} \Phi_{R}^{-\frac{1}{p-1}} d x d t \\
& \quad=C T^{-(\alpha+2) p^{\prime}} \int_{0}^{T} \int_{B_{R}} \omega(t)^{(\beta-\alpha-2) p^{\prime}-\frac{\beta}{p-1}} \Psi_{R}(x)^{\ell} d x d t \\
& \quad \lesssim T^{-(\alpha+2) p^{\prime}+1} R^{n} .
\end{aligned}
$$

We remark that the power of $\omega(t)$ in the integral above is positive, by virtue of (4.5). Let us recall that

$$
(-\triangle)^{\theta} \Psi^{\ell} \leq \ell \Psi^{\ell-1}(-\triangle)^{\theta} \Psi
$$

holds for any $\theta \in(0,1], \ell>1$, and for any nonnegative test function $\Psi$ (see $[2,17])$. Then, since $u$ and $\Psi$ are nonnegative, we may estimate

$$
u(-\triangle)^{\theta} \Psi_{R}(x)^{\ell} \leq \ell u \Psi_{R}(x)^{\ell-1}\left((-\triangle)^{\theta} \Psi_{R}\right)(x) .
$$

It is clear that

$$
\left((-\triangle)^{\theta} \Psi_{R}\right)(x)=R^{-2 \theta}\left((-\triangle)^{\theta} \Psi\right)(x / R),
$$

and that $(-\triangle)^{\theta} \Psi \in \mathcal{S}$, is a bounded function. Therefore, we obtain

$$
\begin{aligned}
& \int_{0}^{T} \int_{B_{R}} u(-\triangle) \varphi d x d t \\
& \quad \leq \ell \int_{0}^{T} \int_{B_{R}} u D_{t \mid T^{\alpha}}^{\alpha} \omega(t)^{\beta} \Psi_{R}(x)^{\ell-1}\left(-\triangle \Psi_{R}\right)(x) d x d t \\
& \quad \leq \epsilon \int_{0}^{T} \int_{B_{R}} u^{p} \Phi_{R} d x d t \\
& \quad+C_{\epsilon} T^{-\alpha p^{\prime}} R^{-2 p^{\prime}} \int_{0}^{T} \int_{B_{R}}\left(D_{t \mid T}^{\alpha} \omega(t)^{\beta} \Psi_{R}(x)^{\ell-1}\right)^{p^{\prime}} \Phi_{R}^{-\frac{1}{p-1}} d x d t .
\end{aligned}
$$

Recalling (4.4) and (4.6), we may estimate the last term by

$$
\begin{aligned}
& T^{-\alpha p^{\prime}} R^{-2 p^{\prime}} \int_{0}^{T} \int_{B_{R}} \omega(t)^{(\beta-\alpha) p^{\prime}-\frac{\beta}{p-1}} \Psi_{R}(x)^{(\ell-1) p^{\prime}-\frac{\ell}{p-1}} d x d t \\
& \quad \leq C T^{-\alpha p^{\prime}+1} R^{-2 p^{\prime}+n} .
\end{aligned}
$$

We notice that the powers of $\beta(t)$ and $\Psi_{R}(x)$ in the integral above are positive, by virtue of $\beta$. Similarly, since $u$ and $-\varphi_{t}$ are nonnegative, we derive 


$$
\begin{aligned}
& -\int_{0}^{T} \int_{\mathbb{R}^{n}} u(-\triangle)^{\frac{1}{2}} \varphi_{t} d x d t \\
& \quad \leq \ell \int_{0}^{T} \int_{B_{R}} u\left(-\partial_{t} D_{t \mid T^{\alpha}}^{\alpha} \omega(t)^{\beta}\right) \Psi_{R}^{\ell-1}\left((-\triangle)^{\frac{1}{2}} \Psi_{R}\right)(x) d x d t \\
& \quad \leq \epsilon \int_{0}^{T} \int_{B_{R}} u^{p} \Phi_{R} d x d t+C_{\epsilon} T^{-(\alpha+1) p^{\prime}+1} R^{-p^{\prime}+n} .
\end{aligned}
$$

We remark that the first passage above is the key point of our approach, since we may restrict the positive part of the integral over $\mathbb{R}^{n}$ to an integral on $B_{R}$ thanks to properties (4.7) and supp $\Psi_{R}=B_{R}$. In other words, even if the fractional Laplacian is a nonlocal operator, we have

$$
\operatorname{supp}\left[(-\triangle)^{\theta} \Psi(x)^{\ell}\right]^{+} \subset \operatorname{supp} \Psi, \quad \text { if } \ell>1 .
$$

This strategy motivated the use of a maximum principle for our problem. Summarizing, we obtained:

$$
\begin{aligned}
& (1-3 \epsilon) \int_{0}^{T} \int_{B_{R}} u^{p} \Phi_{R} d x d t \\
& \quad \leq \bar{C} T^{-\alpha p^{\prime}+1} R^{n}\left(T^{-2 p^{\prime}}+R^{-2 p^{\prime}}+T^{-p^{\prime}} R^{-p^{\prime}}\right) .
\end{aligned}
$$

In the following, we fix $\epsilon \in(0,1 / 3)$. If $p<p_{\gamma}(n)$, it is sufficient to set $R=T$ in (4.8) to derive

$$
\int_{0}^{T} \int_{B_{T}} u^{p} \Phi_{T} d x d t \lesssim T^{-(\alpha+2) p^{\prime}+n+1} .
$$

We notice that $p<p_{\gamma}(n)$ if, and only if, $(\alpha+2) p^{\prime}>n+1$. By Beppo Levi's theorem on monotone convergence, being $\Phi_{T} \nearrow 1$ as $T \rightarrow \infty$, we derive

$$
\int_{0}^{\infty} \int_{\mathbb{R}^{n}} u^{p} d x d t=\lim _{T \rightarrow \infty} \int_{0}^{\infty} \int_{\mathbb{R}^{n}} u^{p} \Phi_{T} d x d t=0 ;
$$

hence $u \equiv 0$.

If $p=p_{\gamma}(n)$, we set $R=T K^{-1}$ for some fixed $K>1$ and from (4.8) we get that

$$
\int_{0}^{T} \int_{B_{T / K}} u^{p} \Phi_{R} d x d t \leq \bar{C} K^{-n}\left(1+K^{p^{\prime}}+K^{2 p^{\prime}}\right),
$$

in particular

$$
\int_{0}^{T} \int_{\mathbb{R}^{n}} u^{p} d x d t=\lim _{T \rightarrow \infty} \int_{0}^{T} \int_{B_{T / K}} u^{p} \Phi_{T / K} d x d t \leq C(K),
$$

for some $C(K)>0$, i.e. $u \in L^{p}$.

On the other hand, $\triangle \Psi_{R}(x)^{\ell}=0$ for $|x| \leq R / 2$, thus we may use Hölder inequality to estimate 


$$
\begin{aligned}
\int_{0}^{T} \int_{\mathbb{R}^{n}} u(-\triangle) \varphi d x d t & =\int_{0}^{T} \int_{|x|>T /(2 K)} u(-\triangle) \varphi d x d t \\
& \leq \int_{0}^{T} \int_{|x|>T /(2 K)} u|\triangle \varphi| d x d t \\
& \lesssim\left(\int_{0}^{T} \int_{|x|>T /(2 K)}|\triangle \varphi|^{p^{\prime}}\left(\Phi_{R}\right)^{-\frac{p^{\prime}}{p}} d x d t\right)^{\frac{1}{p^{\prime}}} \\
& \times\left(\int_{0}^{T} \int_{|x|>T /(2 K)} u^{p} \Phi_{R} d x d t\right)^{\frac{1}{p}}
\end{aligned}
$$

hence, we may replace (4.8) by

$$
\begin{aligned}
& (1-2 \epsilon) \int_{0}^{T} \int_{\mathbb{R}^{n}} u^{p} \Phi_{T / K} d x d t \leq \bar{C} K^{-n}\left(1+K^{p^{\prime}}\right) \\
& +\tilde{C} K^{-\frac{n}{p^{\prime}}+2}\left(\int_{0}^{T} \int_{|x|>T /(2 K)} u^{p} \Phi_{T / K} d x d t\right)^{\frac{1}{p^{\prime}}} .
\end{aligned}
$$

Thanks to $u \in L^{p}$ [see (4.9)], for any fixed $K>1$, it holds

$$
\lim _{T \rightarrow \infty} \int_{0}^{T} \int_{|x|>T /(2 K)} u^{p} \Phi_{T / K} d x d t=0 ;
$$

hence, from (4.10) we derive that

$$
\int_{0}^{\infty} \int_{\mathbb{R}^{n}} u^{p} d x d t \leq \bar{C} K^{-n}\left(1+K^{p^{\prime}}\right)
$$

which is an arbitrarily small quantity, since

$$
p^{\prime}=\frac{n+1}{\alpha+2}<n \text {. }
$$

Therefore, $u \equiv 0$.

If $p<\gamma^{-1}$, i.e. $p^{\prime} \alpha>1$, we set $R=\ln T$ in (4.8) and we apply Beppo Levi's convergence theorem, obtaining

$$
\int_{0}^{\infty} \int_{\mathbb{R}^{n}} u^{p} d x d t=C \lim _{T \rightarrow \infty} T^{-\alpha p^{\prime}+1}(\ln T)^{n}\left(T^{-2 p^{\prime}}+(T \ln T)^{-p^{\prime}}+(\ln T)^{-2 p^{\prime}}\right),
$$

so that $u \equiv 0$ again.

Since $u \equiv 0$ contradicts the assumption of nontrivial data $u_{1}$, we proved our statement.

Remark 4.2. It remains open the question about nonexistence of the global solution only if $p=\gamma^{-1}$ and $\gamma<(n-2) / n$. Indeed, in this case, (4.8) gives

$$
\int_{0}^{\infty} \int_{\mathbb{R}^{n}} u^{p} \Phi_{R} d x d t \leq \bar{C} R^{n-2 p^{\prime}}
$$

but $n>2 p^{\prime}$. In other words, the test function method does not work even if we try to apply it to 


$$
-\triangle u=\int_{0}^{t}(t-s)^{-\gamma}|u(s, x)|^{\frac{1}{\gamma}} d s, \quad 0<\gamma<\frac{n-2}{n} .
$$

We expect that this effect is related to the fact that the test function method does not work for

$$
-\triangle u=|u|^{p}, \quad \text { if } \quad p>\frac{n}{n-2} .
$$

\section{The general case $\sigma \in(0,1]$}

It is natural to ask which critical exponent one may expect for small data solutions to

$$
\left\{\begin{array}{l}
u_{t t}-\triangle u+\mu(-\triangle)^{\sigma} u_{t}=F(t, u), \quad \sigma \in(0,1 / 2), \\
u(0, x)=u_{0}(x) \\
u_{t}(0, x)=u_{1}(x)
\end{array}\right.
$$

taking into account that the critical exponent is given by (1.7) and (1.8) in the two limit cases $\sigma=0$ and $\sigma=1 / 2$.

The linear estimates for (5.1) suggest that the critical exponent should be

$$
\begin{aligned}
\bar{p}(n, \sigma, \gamma) & =\max \left\{p_{\gamma}(n, \sigma), \gamma^{-1}\right\}, \\
p_{\gamma}(n, \sigma) & :=1+\frac{2(2-\sigma-\gamma(1-\sigma))}{[n-2+2 \gamma(1-\sigma)]^{+}} .
\end{aligned}
$$

For $\sigma \in(0,1 / 2)$ the transition from $p_{\gamma}(n, \sigma)$ to $\gamma^{-1}$ happens at $\gamma=$ $(n-2) / n$, for $n \geq 3$, as in the two limit cases $\sigma=0$ and $\sigma=1 / 2$. In particular,

$$
\bar{p}(2, \sigma, \gamma)=p_{\gamma}(2, \sigma)=\frac{2-\sigma}{1-\sigma} \gamma^{-1}
$$

To motivate our expectation, we extend Corollary 2.5 to the following.

Theorem 5.1. Let us consider the following cases:

- $n=1, \sigma \in(0,1 / 4)$ and $1 /(2(1-\sigma))<\gamma<1$,

- $n=2, \sigma \in(0,1 / 2)$ and $\gamma \in(0,1)$.

Let $p>p_{\gamma}(n, \sigma)$. Then there exists $\varepsilon>0$ such that for any initial data as in (2.8), there exists a $\mathcal{C}\left([0, \infty), H^{1}\right) \cap \mathcal{C}^{1}\left([0, \infty), L^{2}\right)$ solution to (5.1). Moreover, the solution satisfies the estimates

$$
\begin{aligned}
&\|u(t, \cdot)\|_{L^{2}} \leq C(1+t)^{1-\left(\frac{n}{4}-\sigma\right) \frac{1}{1-\sigma}-\gamma}\left\|\left(u_{0}, u_{1}\right)\right\|_{\mathcal{A}}, \\
&\|\nabla u(t, \cdot)\|_{L^{2}} \leq \begin{cases}C(1+t)^{1-\left(\frac{3}{4}-\sigma\right) \frac{1}{1-\sigma}-\gamma}\left\|\left(u_{0}, u_{1}\right)\right\|_{\mathcal{A}} & \text { if } n=1, \\
C(1+t)^{-\gamma} \ln (e+t)\left\|\left(u_{0}, u_{1}\right)\right\|_{\mathcal{A}} & \text { if } n=2,\end{cases} \\
&\left\|u_{t}(t, \cdot)\right\|_{L^{2}} \leq C(1+t)^{-\gamma}\left\|\left(u_{0}, u_{1}\right)\right\|_{\mathcal{A} .}
\end{aligned}
$$

Remark 5.2. For the sake of brevity, in Theorem 5.1 we choose to use only estimates on $L^{2}$ basis. Indeed, linear $L^{q_{1}}-L^{q_{2}}$ estimates are more difficult to be derived for (1.4) if $\sigma \in(0,1 / 2)$; see, for instance, [5,6]. Unfortunately, our 
choice imposes the restriction $p \geq 2$, hence we may prove global existence for any $p>\bar{p}(n, \sigma, \gamma)$ and for any $\gamma \in(0,1)$ only in space dimension $n=1,2$.

The model's properties in (1.4) completely change if $\sigma \in(1 / 2,1]$. Indeed, we can prove global existence of small data solutions to

$$
\left\{\begin{array}{l}
u_{t t}-\triangle u+\mu(-\triangle)^{\sigma} u_{t}=F(t, u), \quad \sigma \in(1 / 2,1] \\
u(0, x)=u_{0}(x) \\
u_{t}(0, x)=u_{1}(x)
\end{array}\right.
$$

if $p>\bar{p}(n, \sigma, \gamma)$, with $\bar{p}(n, \sigma, \gamma)$ as in (5.2) and

$$
p_{\gamma}(n, \sigma):=1+\frac{1+2 \sigma(2-\gamma)}{[n-1-2 \sigma(1-\gamma)]^{+}} .
$$

We remark that now the threshold for $\gamma$ such that $p_{\gamma}(n, \sigma)=\gamma^{-1}$ is given by

$$
\gamma=\frac{n-1-2 \sigma}{n}, \quad \text { for } n \geq 3 \text { if } \sigma \in(1 / 2,1) \text { and for } n \geq 4 \text { if } \sigma=1 \text {. }
$$

Theorem 5.3. Let us consider the following cases:

- $n=2, \sigma \in(1 / 2,1], \gamma \in((2 \sigma-1) /(2 \sigma), 1)$ and $p>p_{\gamma}(2, \sigma)$,

- $n=3, \sigma \in(1 / 2,3 / 4), \gamma \in((3-4 \sigma) / 3,1)$ and $p \in(\bar{p}(3, \sigma, \gamma), 3 /(3-4 \sigma)]$,

- $n=3, \sigma \in[3 / 4,1], \gamma \in(0,1)$ and $p>\bar{p}(3, \sigma, \gamma)$,

- $n=4, \sigma=1, \gamma \in(0,1)$ and $p>\bar{p}(4,1, \gamma)$.

Then there exists $\varepsilon>0$ such that for any initial data

$$
\left(u_{0}, u_{1}\right) \in \mathcal{A}:=\left(L^{1} \cap H^{2 \sigma}\right) \times\left(L^{1} \cap L^{2}\right) \quad \text { with }\left\|\left(u_{0}, u_{1}\right)\right\|_{\mathcal{A}} \leq \varepsilon,
$$

there exists a $\mathcal{C}\left([0, \infty), H^{2 \sigma}\right) \cap \mathcal{C}^{1}\left([0, \infty), L^{2}\right)$ solution to (5.4). Moreover, the solution satisfies the estimates

$$
\begin{aligned}
&\|u(t, \cdot)\|_{L^{2}} \leq C(1+t)^{1-\frac{n-2}{4 \sigma}-\gamma}\left\|\left(u_{0}, u_{1}\right)\right\|_{\mathcal{A}}, \\
& \mathcal{E}[u](t) \leq \begin{cases}C(1+t)^{1-\frac{1}{2 \sigma}-\gamma}\left\|\left(u_{0}, u_{1}\right)\right\|_{\mathcal{A}} & \text { if } n=2, \\
C(1+t)^{1-\frac{3}{4 \sigma}-\gamma}\left\|\left(u_{0}, u_{1}\right)\right\|_{\mathcal{A}} & \text { if } n=3, \sigma \in(3 / 4,1), \\
C(1+t)^{-\gamma} \ln (e+t)\left\|\left(u_{0}, u_{1}\right)\right\|_{\mathcal{A}} & \text { if } n=3, \sigma=3 / 4, \\
C(1+t)^{-\gamma}\left\|\left(u_{0}, u_{1}\right)\right\|_{\mathcal{A}} & \text { if } n=3, \sigma \in(1 / 2,3 / 4), \\
C(1+t)^{-\gamma} \ln (e+t)\left\|\left(u_{0}, u_{1}\right)\right\|_{\mathcal{A}} & \text { if } n=4 \text { and } \sigma=1,\end{cases} \\
&\|u(t, \cdot)\|_{\dot{H}^{2 \sigma}} \leq \begin{cases}C(1+t)^{-\gamma} \ln (e+t)\left\|\left(u_{0}, u_{1}\right)\right\|_{\mathcal{A}} & \text { if } n=2, \\
C(1+t)^{-\gamma}\left\|\left(u_{0}, u_{1}\right)\right\|_{\mathcal{A}} & \text { if } n \geq 3 .\end{cases}
\end{aligned}
$$

The limit case $\sigma=1$ has a particular interest, since in the damping term in (5.7) it appears an integer power of Laplacian; hence, we may use the test function method to state the following result. 
Theorem 5.4. Let us consider Cauchy problem

$$
\left\{\begin{array}{l}
u_{t t}-\Delta u-\mu \Delta u_{t}=\int_{0}^{t}(t-s)^{-\gamma}|u(s, x)|^{p} d s \\
u(0, x)=u_{0}(x) \\
u_{t}(0, x)=u_{1}(x)
\end{array}\right.
$$

and

with $\mu>0, \gamma \in(0,1), p>1$ and initial data satisfying $-\triangle u_{0}+u_{1} \in L^{1}$

$$
\int_{\mathbb{R}^{n}}\left(-\triangle u_{0}(x)+u_{1}(x)\right) d x>0 .
$$

Then, there exists no global weak solution to (5.7) in the following four cases:

- if $n=1$, for any $\gamma \in(0,1)$ and $p>1$,

- if $n \geq 2$, for any $\gamma \in[(n-2) / n, 1)$ and $p \in\left(1, p_{\gamma}(n)\right]$,

- if $n \geq 3$, for any $\gamma \in(0,(n-2) / n)$ and $p \in\left(1, \gamma^{-1}\right)$.

We remark that the nonexistence exponent in Theorem 5.4 is the same obtained in the case $\sigma=1 / 2$ in Theorem 2.11. Therefore, we did not prove the optimality of $\bar{p}(n, 1, \gamma)$, in general, since the nonempty interval

$$
(\bar{p}(n, \gamma), \bar{p}(n, 1, \gamma)]
$$

is not covered by Theorem 5.3 neither by Theorem 5.4 , for $\gamma \in([n-$ $\left.3]^{+} / n, 1\right)$.

However, if $n \geq 4$ and $\gamma \in(0,(n-3) / n]$, it holds $\bar{p}(n, 1, \gamma)=\bar{p}(n, \gamma)=$ $\gamma^{-1}$, that is, the influence of the nonlinear memory on (1.4) is so strong that the global existence exponent is the same for any $\sigma \in[0,1]$. In particular, if $n=4$ and $\gamma \in(0,1 / 4]$, combining Theorems 5.3 and 5.4 , we showed that the critical exponent $\gamma^{-1}$ is optimal.

We now prove our statements.

Proof of Theorem 5.1. In [8] it has been proved that the solution to

$$
\left\{\begin{array}{l}
v_{t t}-\triangle v+\mu(-\triangle)^{\sigma} v_{t}=0, \quad \sigma \in(0,1 / 2), \\
v(0, x)=v_{0}(x) \\
v_{t}(0, x)=v_{1}(x)
\end{array}\right.
$$

satisfies the estimates

$$
\begin{aligned}
\|v(t, \cdot)\|_{L^{2}} & \leq(1+t)^{-\left(\frac{n}{4}-\sigma\right) \frac{1}{1-\sigma}}\left\|\left(v_{0}, v_{1}\right)\right\|_{L^{1} \cap L^{2}} \\
\|\nabla v(t, \cdot)\|_{L^{2}} & \leq(1+t)^{-\left(\frac{n+2}{4}-\sigma\right) \frac{1}{1-\sigma}}\left(\left\|v_{0}\right\|_{L^{1} \cap H^{1}}+\left\|v_{1}\right\|_{L^{1} \cap L^{2}}\right), \\
\left\|v_{t}(t, \cdot)\right\|_{L^{2}} & \leq(1+t)^{-\left(\frac{n}{4}-\sigma\right) \frac{1}{1-\sigma}-1}\left(\left\|v_{0}\right\|_{L^{1} \cap H^{1}}+\left\|v_{1}\right\|_{L^{1} \cap L^{2}}\right)
\end{aligned}
$$


provided that $\sigma \in(0,1 / 4)$ if $n=1$. We follow the proof of Theorem 2.3. For a fixed $T>0$, let

$$
\begin{aligned}
X(T):= & \mathcal{C}\left([0, T], H^{1}\right) \cap \mathcal{C}^{1}\left([0, T], L^{2}\right), \\
\|w\|_{X(T)}:= & \max _{t \in[0, T]}\left\{(1+t)^{-1+\left(\frac{n}{4}-\sigma\right) \frac{1}{1-\sigma}+\gamma}\|w(t, \cdot)\|_{L^{2}}\right. \\
& \left.+f(t)\|\nabla w(t, \cdot)\|_{L^{2}}+(1+t)^{\gamma}\left\|w_{t}(t, \cdot)\right\|_{L^{2}}\right\},
\end{aligned}
$$

where

$$
f(t)= \begin{cases}(1+t)^{-1+\left(\frac{3}{4}-\sigma\right) \frac{1}{1-\sigma}+\gamma} & \text { if } n=1 \\ (1+t)^{\gamma} \ell(t)^{-1} & \text { if } n=2 .\end{cases}
$$

We recall that $\ell(t):=\ln (e+t)$. Since $p>p_{\gamma}(n, \sigma)>2$ for $n=1,2$, by Gagliardo-Nirenberg inequality (3.5), it follows from (5.11) that

$$
\|w(t, \cdot)\|_{L^{p} \cap L^{2 p}} \leq \begin{cases}C(1+t)^{1-\left(\frac{1}{2}\left(1-\frac{1}{p}\right)-\sigma\right) \frac{1}{1-\sigma}-\gamma}\|w\|_{X(T)} & \text { if } n=1 \\ C(1+t)^{1-\left(1-\frac{1}{p}-\sigma\right) \frac{1}{1-\sigma}-\gamma} \ell(t)\|w\|_{X(T)} & \text { if } n=2 .\end{cases}
$$

Therefore,

$$
\|w(t, \cdot)\|_{L^{p} \cap L^{2 p}}^{p} \leq(1+t)^{-\beta}\|w\|_{X(T)}^{p},
$$

for some $\beta>1$ if, and only if, $\mathrm{y}$

$$
p\left(\left(\frac{n}{2}\left(1-\frac{1}{p}\right)-\sigma\right) \frac{1}{1-\sigma}+\gamma-1\right)>1
$$

that is, $p>p_{\gamma}(n, \sigma)$. By Duhamel's principle, for $j+k=0,1$ we derive $\left\|\nabla^{j} \partial_{t}^{k} G w(t, \cdot)\right\|_{L^{2}}$

$$
\leq C\|w\|_{X(T)} \int_{0}^{t}(1+t-\tau)^{-\left(\frac{n+2 j}{4}-\sigma\right) \frac{1}{1-\sigma}-k} \int_{0}^{\tau}(\tau-s)^{-\gamma}(1+s)^{-\beta} d s d \tau .
$$

Applying Lemma 3.2, we prove (3.14). Similarly, we prove (3.15). The global existence of the solution follows and we may derive the desired estimates as in the proof of Theorem 2.3.

Proof of Theorem 5.3. The solution to

$$
\left\{\begin{array}{l}
v_{t t}-\triangle v+\mu(-\triangle)^{\sigma} v_{t}=0, \quad \sigma \in(1 / 2,1] \\
v(0, x)=v_{0}(x) \\
v_{t}(0, x)=v_{1}(x)
\end{array}\right.
$$

satisfies the following estimates (see [26] for $\sigma=1$ and [8] for $\sigma \in$ $(1 / 2,1))$ :

$$
\begin{aligned}
\|v(t, \cdot)\|_{L^{2}} & \leq(1+t)^{-\frac{n-2}{4 \sigma}}\left\|\left(v_{0}, v_{1}\right)\right\|_{L^{1} \cap L^{2}}, \\
\mathcal{E}[v](t) & \leq(1+t)^{-\frac{n}{4 \sigma}}\left(\left\|v_{0}\right\|_{L^{1} \cap H^{1}}+\left\|v_{1}\right\|_{L^{1} \cap L^{2}}\right), \\
\|v(t, \cdot)\|_{\dot{H}^{2 \sigma}} & \leq(1+t)^{-\frac{n-2}{4 \sigma}-1}\left(\left\|v_{0}\right\|_{L^{1} \cap H^{2 \sigma}}+\left\|v_{1}\right\|_{L^{1} \cap L^{2}}\right) .
\end{aligned}
$$


First, let $n=2$. We may follow the proof of Theorem 5.1, setting

$$
\begin{aligned}
X(T):= & \mathcal{C}\left([0, T], H^{2 \sigma}\right) \cap \mathcal{C}^{1}\left([0, T], L^{2}\right), \\
\|w\|_{X(T)}:= & \max _{t \in[0, T]}\left\{(1+t)^{-1+\gamma}\|w(t, \cdot)\|_{L^{2}}\right. \\
& \left.+(1+t)^{-1+\frac{1}{2 \sigma}+\gamma} \mathcal{E}[w](t)+(1+t)^{\gamma} \ell(t)^{-1}\|w(t, \cdot)\|_{\dot{H}^{2 \sigma}}\right\} .
\end{aligned}
$$

Since $p>p_{\gamma}(2, \sigma)>2$, we may use Gagliardo-Nirenberg inequality (3.5) to derive that

$$
\|w(t, \cdot)\|_{L^{p} \cap L^{2 p}} \leq C(1+t)^{1+\frac{1}{2 \sigma}-\frac{1}{\sigma}\left(1-\frac{1}{p}\right)-\gamma} \ell(t)\|w\|_{X(T)} .
$$

Therefore,

$$
\|w(t, \cdot)\|_{L^{p} \cap L^{2 p}}^{p} \leq(1+t)^{-\beta}\|w\|_{X(T)}^{p},
$$

for some $\beta>1$ if, and only if, $p>p_{\gamma}(2, \sigma)$. The end of the proof follows. If $n=3,4$, we should proceed in a different way, employing fractional Sobolev embeddings instead of Gagliardo-Nirenberg inequality (3.5), as in [4]. We notice that the solution to (5.12) satisfies the estimate:

$\|v(t, \cdot)\|_{\dot{H}^{k}} \leq(1+t)^{-\frac{n+2 k-2}{4 \sigma}}\left(\left\|v_{0}\right\|_{L^{1} \cap H^{2 \sigma}}+\left\|v_{1}\right\|_{L^{1} \cap L^{2}}\right), \quad$ for any $k \in[0,2 \sigma]$.

Let $n=3$ and let us denote $\kappa=\kappa(\sigma):=2 \sigma-1 / 2$. Let $X(T)$ be as in (5.13), with norm given by

$$
\begin{aligned}
\|w\|_{X(T)}:= & \max _{t \in[0, T]}\left\{(1+t)^{-1+\frac{1}{4 \sigma}+\gamma}\|w(t, \cdot)\|_{L^{2}}+f_{\sigma}(t) \mathcal{E}[w](t)\right. \\
& \left.+(1+t)^{\gamma}\left(\ell(t)^{-1}\|w(t, \cdot)\|_{\dot{H}^{\kappa}}+\|w(t, \cdot)\|_{\dot{H}^{2 \sigma}}\right)\right\},
\end{aligned}
$$

where

$$
f_{\sigma}(t)= \begin{cases}(1+t)^{\gamma} & \text { if } \sigma \in(1 / 2,3 / 4), \\ (1+t)^{\gamma} \ell(t)^{-1} & \text { if } \sigma=3 / 4 \\ (1+t)^{-1+\frac{3}{4 \sigma}+\gamma} & \text { if } \sigma \in(3 / 4,1] .\end{cases}
$$

For any $p \geq 2$ we may use the fractional Sobolev embedding $\|w(t, \cdot)\|_{L^{p} \cap L^{2 p}} \leq C\|w(t, \cdot)\|_{\dot{H}^{k_{1} \cap \dot{H}^{k_{2}}}}, \quad$ with $k_{m}:=\frac{n}{2}\left(1-\frac{1}{m p}\right) ;$

hence, we derive

$$
\|w(t, \cdot)\|_{L^{p} \cap L^{2 p}} \leq C(1+t)^{-\gamma+\left[1+\frac{1}{2 \sigma}-\frac{3}{2 \sigma}\left(1-\frac{1}{p}\right)\right]^{+}} \ell(t)\|w\|_{X(T)} .
$$

for any $p>\bar{p}(3, \sigma, \gamma)$, provided that $p<3 /(3-4 \sigma)$ if $\sigma \in(1 / 2,3 / 4)$. The end of the proof follows.

In the last case, $n=4$ and $\sigma=1$, we proceed as for $n=3$, but we take

$$
\begin{aligned}
\|w\|_{X(T)}:= & \max _{t \in[0, T]}\left\{(1+t)^{-\frac{1}{2}+\gamma}\|w(t, \cdot)\|_{L^{2}}\right. \\
& \left.+(1+t)^{\gamma}\left(\ell(t)^{-1} \mathcal{E}[w](t)+\left\|\nabla^{2} w(t, \cdot)\right\|_{L^{2}}\right)\right\} .
\end{aligned}
$$

This concludes the proof. 
Proof of Theorem 5.4. The proof is similar to the proof of Theorem 2.11 but, due to the fact that the damping term does not contain fractional powers of the Laplacian, we do not need a maximum principle for (5.7).

We may write

$$
\begin{aligned}
& \int_{0}^{T} \int_{B_{R}} u\left(\varphi_{t t}-\triangle \varphi\right) d x d t+\mu \int_{0}^{T} \int_{B_{R}} u \triangle \varphi_{t} d x d t \\
& \quad=\int_{B_{R}}\left(-\mu \triangle u_{0}(x)+u_{1}(x)\right) \varphi(0, x) d x+\Gamma(\alpha) \int_{0}^{T} \int_{B_{R}} J_{0 \mid t}^{\alpha}\left(|u|^{p}\right) \varphi d x d t .
\end{aligned}
$$

Thanks to (5.8), we derive

$$
-\int_{B_{R}}\left(-\triangle u_{0}(x)+u_{1}(x)\right) \varphi(0, x) d x \leq 0
$$

for any sufficiently large $R>0$. We follow the proof of Theorem 2.11 to estimate the term containing $\varphi_{t t}-\triangle \varphi$. Indeed, it still holds

$$
\int_{0}^{T} \int_{B_{R}}|u| \varphi_{t t} d x d t \leq \epsilon \int_{0}^{T} \int_{B_{R}}|u|^{p} \Phi_{R} d x d t+C_{\epsilon} T^{-(\alpha+2) p^{\prime}+1} R^{n},
$$

as well as

$$
\int_{0}^{T} \int_{B_{R}}|u||\triangle \varphi| d x d t \leq \epsilon \int_{0}^{T} \int_{B_{R}}|u|^{p} \Phi_{R} d x d t+C_{\epsilon} T^{-\alpha p^{\prime}+1} R^{-2 p^{\prime}+n},
$$

whereas the contribution coming from the damping term may now be directly estimated as

$$
\int_{0}^{T} \int_{\mathbb{R}^{n}}|u|\left|\triangle \varphi_{t}\right| d x d t \leq \epsilon \int_{0}^{T} \int_{B_{R}}|u|^{p} \Phi_{R} d x d t+C_{\epsilon} T^{-(\alpha+1) p^{\prime}+1} R^{-2 p^{\prime}+n} .
$$

Summarizing, we obtained

$$
(1-3 \epsilon) \int_{0}^{T} \int_{B_{R}} u^{p} \Phi_{R} d x d t \leq \bar{C} T^{-\alpha p^{\prime}+1} R^{n}\left(T^{-2 p^{\prime}}+R^{-2 p^{\prime}}+T^{-p^{\prime}} R^{-2 p^{\prime}}\right),
$$

which is equivalent to (4.8). Therefore, by taking $T$ and $R$ as in the proof of Theorem 2.11, it follows $u \equiv 0$ for any $p<\bar{p}(n, \gamma)$. If $p=p_{\gamma}(n)$, we proceed again as in the proof of Theorem 2.11.

The proof of the statement follows from the fact that $u \equiv 0$ contradicts $(5.8)$.

\section{References}

[1] Charão, R.C., da Luz, C.R., Ikehata, R.: Sharp decay rates for wave equations with a fractional damping via new method in the fourier space. J. Math. Anal. Appl. 408(1), 247-255 (2013). doi:10.1016/j.jmaa.2013.06.016

[2] Córdoba, A., Córdoba, D.: A maximum principle applied to quasigeostrophic equations. Commun. Math. Phys. 249, 511-528 (2004). doi:10.1007/ s00220-004-1055-1 
[3] Cui, S.: Local and global existence of solutions to semilinear parabolic initial value problems. Nonlinear Anal. 43, 293-323 (2001)

[4] D'Abbicco, M.: The influence of a nonlinear memory on the damped wave equation. Nonlinear Anal. 95, 130-145 (2014). doi:10.1016/j.na.2013.09.006

[5] D'Abbicco, M., Ebert, M.R: Diffusion phenomena for the wave equation with structural damping in the $L^{p}-L^{q}$ framework. J. Differ. Equ. 256, 2307-2336 (2014). doi:10.1016/j.jde.2014.01.002

[6] D'Abbicco, M., Ebert, M.R: An application of Lp-Lq decay estimates to the semilinear wave equation with parabolic-like structural damping. Nonlinear Anal. 99, 16-34 (2014). doi:10.1016/j.na.2013.12.021

[7] D'Abbicco, M., Lucente, S., Reissig, M.: Semilinear wave equations with effective damping. Chin. Ann. Math., Series B. 34(3), 345-380 (2013). doi:10.1007/ s11401-013-0773-0

[8] D'Abbicco, M., Reissig, M.: Semilinear structural damped waves. Math. Methods Appl. Sci. (2013). doi:10.1002/mma.2913

[9] D'Ancona, P., Spagnolo, S.: Nonlinear perturbations of the Kirchhoff equation. Commun. Pure Appl. Math. 47, 1005-1029 (1993)

[10] Fang, D., Lu, X., Reissig, M.: High-order energy decay for structural damped systems in the electromagnetical field. Chin. Ann. Math. 31(2), 237-246 (2010). doi:10.1007/s11401-008-0185-8

[11] Fino, A.: Critical exponent for damped wave equations with nonlinear memory. Nonlinear Anal. 74, 5495-5505 (2011)

[12] Ghisi, M., Gobbino, M., Haraux, A.: Local and global smoothing effects for some linear hyperbolic equations with a strong dissipation. arXiv:1402.6595 [math.AP] (2014)

[13] Ikehata, R.: Asymptotic profiles for wave equations with strong damping. arXiv: 1402.6073 [math.AP] (2014)

[14] Ikehata, R., Natsume, M.: Energy decay estimates for wave equations with a fractional damping. Differ. Integral Equ. 25(9-10), 939-956 (2012)

[15] Ikehata, R., Tanizawa, K.: Global existence of solutions for semilinear damped wave equations in $R^{N}$ with noncompactly supported initial data. Nonlinear Anal. 61, 1189-1208 (2005)

[16] Ikehata, R., Todorova, G., Yordanov, B.: Wave equations with strong damping in Hilbert spaces. J. Differ. Equ. 254, 3352-3368 (2013). doi:10.1016/j.jde.2013. 01.023

[17] Ju, N.: The maximum principle and the global attractor for the dissipative 2D quasi-geostrophic equations. Commun. Math. Phys. 255, 161-181 (2005). doi:10. 1007/s00220-004-1256-7 
[18] Kobayashi, T., Shibata, Y.: Remark on the rate of decay of solutions to linearized compressible Navier-Stokes equations. Pac. J. Math. 207(1), 199-234 (2002)

[19] Lu, X., Reissig, M.: Rates of decay for structural damped models with decreasing in time coefficients. Int. J. Dyn. Syst. Differ. Equ. 2, 21-55 (2009). doi:10.1504/ IJDSDE.2009.028034

[20] Lucente, S., Ziliotti, G.: A decay estimate for a class of hyperbolic pseudodifferential equations. Rend. Mat. Acc. Lincei s. 10(9), 173-190 (1999)

[21] Narazaki, T.: $L^{p}-L^{q}$ estimates for damped wave equations and their applications to semilinear problem. J. Math. Soc. Jpn. 56, 586-626 (2004)

[22] Narazaki, T., Reissig, M.: $L^{1}$ estimates for oscillating integrals related to structural damped wave models. In: Cicognani, M., Colombini, F., Del Santo, D. (eds.) Studies in Phase Space Analysis with Applications to PDEs. Progress in Nonlinear Differential Equations and Their Applications, pp. 215-258. Birkhäuser, Boston (2013)

[23] Podlubny, I.: Fractional Differential Equations. Math. Sci. Eng. vol. 198. Academic Press, San Diego. ISBN 0-12 55840-2

[24] Reissig, M.: Rates of decay for structural damped models with strictly increasing in time coefficients. Contemp. Math. 554, 187-206 (2011). Israel Mathematical Conference Proceedings, Complex Analysis and Dynamical Systems IV, Part 2. May 18-22, Nahariya, Israel

[25] Samko, S.G., Kilbas, A.A., Marichev, O.I.: Fractional integrals and derivatives, theory and applications. Gordon and Breach Science Publishers, USA (1987)

[26] Shibata, Y.: On the rate of decay of solutions to linear viscoelastic equation. Math. Methods Appl. Sci. 23, 203-226 (2000)

[27] Todorova, G., Yordanov, B.: Critical exponent for a nonlinear wave equation with damping. J. Differ. Equ. 174, 464-489 (2001)

Marcello D'Abbicco

Department of Mathematics

University of Bari

Via E. Orabona 4

70125 Bari

Italy

e-mail: m.dabbicco@gmail.com

Received: 5 November 2013.

Accepted: 16 February 2014. 